\title{
WSRT and Lofar observations of the Lockman Hole
}

\author{
Gabriele Guglielmino \\ Università di Bologna, ITALY; IRA-INAF, Bologna, ITALY; ASTRON, The Netherlands \\ Via gobetti 101, Bologna, Italy \\ E-mail: guglielm@ira.inaf.it
}

\section{Isabella Prandoni}

IRA-INAF, Bologna, ITALY

\section{Raffaella Morganti}

ASTRON, the Netherlands Institute for Radio Astronomy, Postbus 2, 7990 AA, Dwingeloo, The

Netherlands.; Kapteyn Astronomical Institute, University of Groningen, P.O. Box 800,

9700 AV Groningen, The Netherlands

\section{George Heald}

ASTRON, the Netherlands Institute for Radio Astronomy, Postbus 2, 7990 AA, Dwingeloo, The Netherlands.

\begin{abstract}
The nature and evolutionary properties of the faint radio population, responsible for the steepening observed in the $1.4 \mathrm{GHz}$ source counts below $1 \mathrm{mJy}$, are not yet completely understood. We know that is a mixture of star-forming galaxies and high-redshift and/or lowluminosity AGN, but the relative importance of the various classes of objects is still matter of debate. Multi-wavelength information and radio spectral indices may help to constrain the origin of the radio emission in such faint radio sources, and may be fundamental in understanding possible links to the bolometric emission of the host galaxies. With the aim of studying the physical properties of the faint radio population, we are performing a multi-frequency radio analysis of the sources in a well-known deep field: the Lockman Hole, which is one of the best studied sky regions in different wavebands. This will provide us with important complementary data (for example redshifts) to the radio data, allowing us to characterize the physical and evolutionary properties of the various classes of sources composing the faint radio population. The Lockman Hole was observed with Westerbork at $1.4 \mathrm{GHz}$ and $325 \mathrm{MHz}$, and with Lofar at $150 \mathrm{MHz}$ as part of the commissioning of the telescope. Lofar imaging of the Lockman Hole can play an important role in this project, allowing, for the very first time, to observe the submJy source population at very low frequencies (30-200 MHz), where self-absorption phenomena are expected to be very important. The rms noise reached in these observations is of the order of $11 \mu \mathrm{Jy}, 0.6 \mathrm{mJy}$ and $1.5 \mathrm{mJy}$, respectively. We present preliminary results of all these radio observations.
\end{abstract}




\section{Introduction}

In early studies, radio astronomy was limited to bright sources associated with rare luminous $\left(L_{1,4 G H z} \approx 10^{25-29} \mathrm{WHz}^{-1}\right)$ radio galaxies and quasars [quasi-stellar objects (QSOs)]. Galaxies with nuclear activity are usually characterized by powerful radio lobes, which are evidence of interactions between highly collimated relativistic flows, produced by the nuclear activity, and the interstellar/ intergalactic medium. These magnificent radio-loud structures were classified by Fanaroff \& Riley (1974) depending on their shape (FR I and II classes), and optical identifications showed that these active galactic nuclei (AGN) are usually hosted by massive elliptical galaxies. It was not until the 1980s that radio source counts at the sub-mJy level revealed a new radio population (Windhorst et al. 1985; Mitchell \&Condon 1985). The nature of the faint radio sources which dominate the number counts below $\sim 1 \mathrm{mJy}$ is controversial. Various studies (e.g. Simpson et al. 2006; Seymour et al. 2008; Smolcic et al. 2008; Mignano et al. 2008; Prandoni et al. 2010) have identified this population with star-forming galaxies (starbursts, spirals or irregulars) and low luminosity radio AGN (faint FR I, Seyfert galaxies).

The relative contribution of low-power/high-z AGN and star-forming galaxies to the sub-mJy radio regime are still hotly debated. Many different approaches have been taken to disentangle these two populations: using far-infrared (far-IR)/radio flux ratios; tackling their radio brightness temperatures and luminosities; resolving their radio morphologies; identifying optical host galaxies via morphology, or spectroscopy, or their locus in colour-colour diagrams, via Xray identifications. These approaches tend to yield substantially different results. Understanding whether the dominant triggering process is star formation or nuclear activity has important implications on the study of the star formation/black hole accretion history with radio-selected samples. In this respect an important piece of evidence may come from multi-frequency radio observations. Radio spectral indices may help to constrain the origin of the radio emission in faint radio sources and may actually be fundamental for understanding eventual links to the optical light.

In terms of spectral indices, $\alpha$, star-forming galaxies (SFG) are usually considered to have a mean spectral index between -0.8 and -0.7 (where $S \propto v^{\alpha}$ ), with a relatively small dispersion, 0.24 . Nearby $(\mathrm{z}<0.5)$ FR I \& II radio galaxies has also been found to have similar spectral indexes (between 178 and $750 \mathrm{MHz}$ ), with mean and scatter of $\alpha=0.74 \pm 0.19$ and $\alpha=0.79 \pm$ 0.14 , respectively. This implies that studies based on the radio spectral index have large difficulties disentangling star-forming from steep-spectrum radio galaxy populations. Nevertheless, the radio spectral index is sensitive to core-dominated AGN, GHz-peaked sources (GPS) and to ultra-steep spectrum sources (USS) usually found at high redshift.

Recent studies (e.g. Prandoni et al 2006; Bondi et al. 2007; Garn et al. 2008, Magliocchetti et al. 2008), combining 1.4 and $5 \mathrm{GHz}$ or $610 \mathrm{MHz}$ and $1.4 \mathrm{GHz}$ data, have found evidence for flatter 
spectral indices and larger dispersions at sub-mJy radio fluxes, suggesting that core-dominated AGN are playing a key role in the sub-mJy radio population.

Low frequency observations are particularly important to investigate the flattening of the spectral index distribution of the sub-mJy radio population, because possible synchrotron selfabsorbed mechanism become more prominent with decreasing frequency.

The LOw Frequency Array (LOFAR) is a next-generation radio telescope operating at 30-200 $\mathrm{MHz}$. The large collecting area and instantaneous fractional bandwidth, together with long baselines, allow the first detailed study of the sub-mJy source population below $200 \mathrm{MHz}$.

This work presents two different WSRT observations of the Lockman Hole: a deep radio mosaic obtained at $1.4 \mathrm{GHz}$ and a $325 \mathrm{MHz}$ image. We are performing deep Lofar observations of the Lockman Hole, to extend our analysis to lower frequency (150 MHz). These we will present our first $150 \mathrm{MHz}$ image obtained in the framework of the Lofar commissioning.

\subsection{WSRT Observations: $1.4 \mathrm{GHz}$ and $325 \mathrm{MHz}$}

The Lockman Hole was observed with the Westerbork Synthesis Radio Telescope (WSRT) at $1.4 \mathrm{GHz}$. The observations covered an area of 6.6 square degrees, with a mosaic of 16 overlapping pointings. The synthesized beam is 11 " $\mathrm{x} 9$ " and the rms was found to be approximately uniform over the central 2 sq. degr. region (shown in Figure 1), with a value of about $11 \mu \mathrm{Jy}$. This is the very first time that such a large area was imaged to such depth, allowing a detailed statistical study of the sub-mJy population, down to $\mu J y$ levels. The extracted $1.4 \mathrm{GHz}$ catalog lists about 6000 sources, $90 \%$ of them being sub-mJy sources. This is one of the largest $\mu \mathrm{Jy}$ samples available so far. We used all sources brighter than $70 \mu \mathrm{Jy}$ (corresponding to $\sim 7 \sigma$ in the inner part of the mosaic) to derive the differential source counts as a function of flux density (see Figure 2). Such counts are consistent with most of those reported in the literature, and provide one of the most statistically robust determinations in the flux range $0.1<\mathrm{S}<1 \mathrm{mJy}$ (Guglielmino et al. 2012).

In January 2012, the same region imaged at $1.4 \mathrm{GHz}$, was observed at $325 \mathrm{MHz}$. Due to the limited resolution of the WSRT telescope at this frequency ( 40x70 arcsec), the image obtained is confusion-limited at a level of $\sim 0.6-0.7 \mathrm{mJy}$ (rms). Such data will be useful to study the radio spectral behaviour of mJy radio sources in the Lockman Hole field.

\subsection{Lofar Observations at $150 \mathrm{MHz}$}

The Lockman Hole was observed with Lofar (20 core and 9 remote stations) at $150 \mathrm{MHz}$ in October 2011, as part of the commissioning of the telescope. This $6 \mathrm{~h}$ observation was performed with one beam centered on the bright source 3C244.1, which is located at $\sim 3$ degrees from the center of the Lockman Hole field; a second beam was centered on the Lockman Hole itself. In addition the calibrator 3C196 was separately observed every 30min for one minute. The total bandwidth is $24 \mathrm{MHz}$. This setup allowed a good modeling of the 3C244.1 source, and, as a result, a good calibration of the entire Lockman Hole field. The Lofar reduction pipeline was used for data flagging and calibration. We used the CASA data reduction software 
for the deconvolution. The image obtained from 60 sub-bands is shown in Figure 3. The synthesized beam is 8 " $\mathrm{x} 4$ " and the noise reached is $1.5 \mathrm{mJy}(\mathrm{rms})$.

\subsection{Radio spectral analysis and Primary Beam Correction}

Several low frequency counterparts of the brightest WSRT $1.4 \mathrm{GHz}$ sources are already clearly visible in the Lofar image (see examples in Figure 4). This allows us to start investigating the low-frequency spectral index properties of sources brighter than 15-20 mJy. This limit is significantly deeper than what allowed by the existing 7C $151 \mathrm{MHz}$ survey (flux limit $70 \mathrm{mJy}$, completeness limit $200 \mathrm{mJy}$ ).

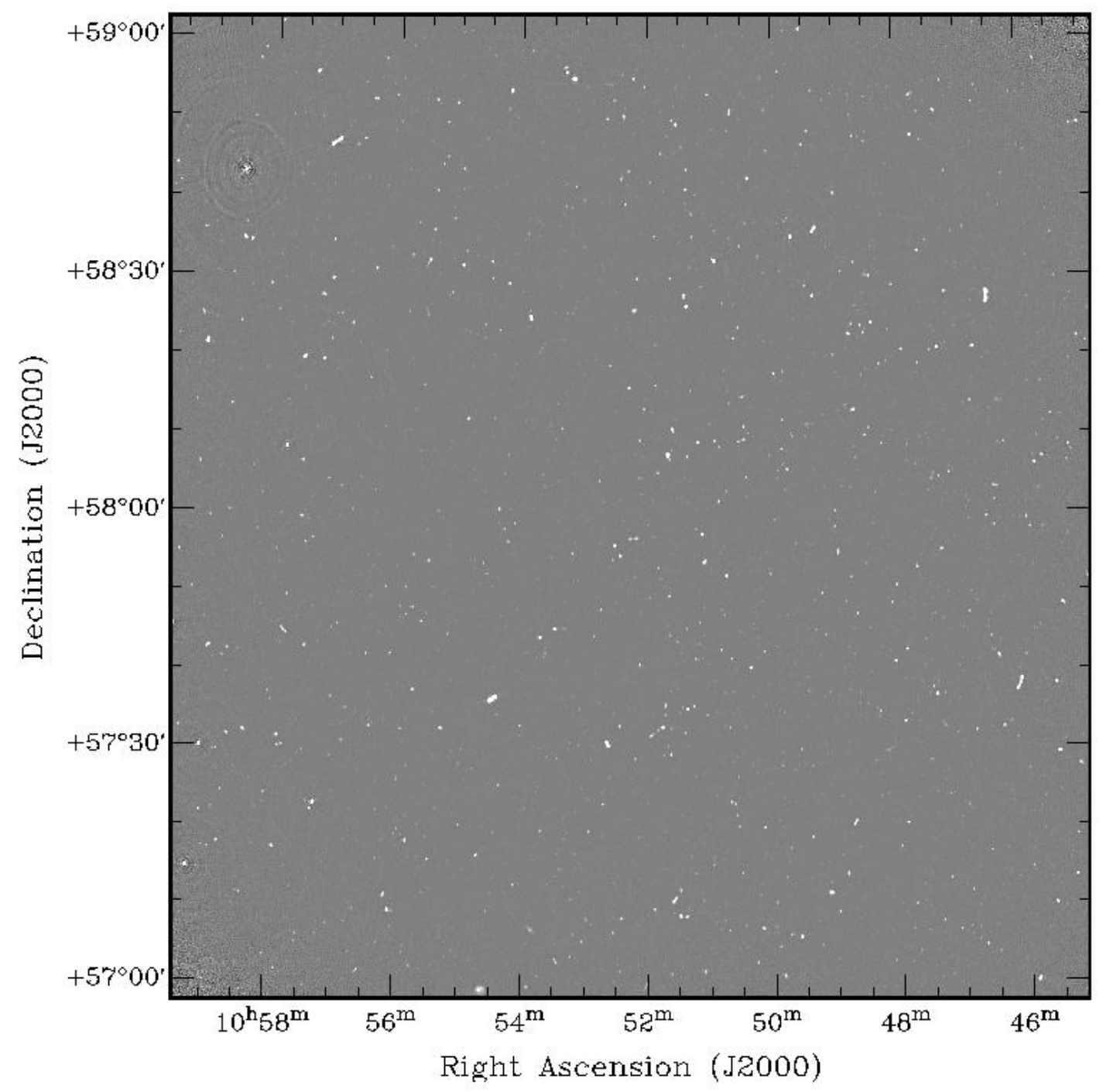

Figure 1 The inner part ( 2 sq. degr.) of the WSRT $1.4 \mathrm{GHz}$ mosaic 


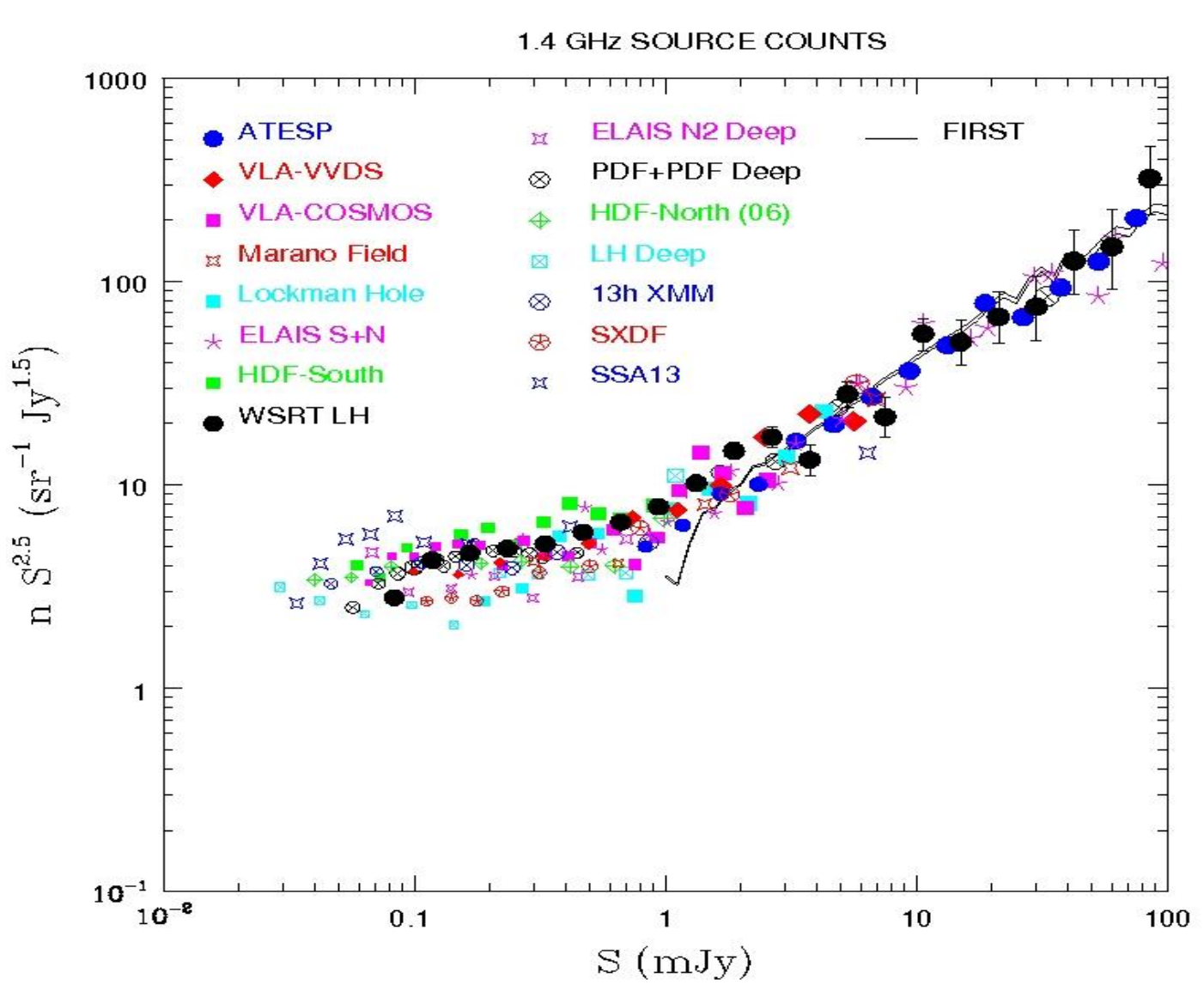

Figure 2 Normalized $1.4 \mathrm{GHz}$ differential source counts obtained from our sample (black filled circles) compared with counts obtained from other samples (as indicated in the figure)

To perform this analysis on the entire field (and not only in the very center of it), we need, however, to correct the $150 \mathrm{MHz}$ image fluxes for the Lofar primary beam response, which unfortunately is not yet fully understood in the HBA band. This is necessary to correct for the source flux density attenuation introduced by the primary beam, as we move toward the external parts of the $150 \mathrm{MHz}$ image.

We have used the brightest sources in the sample (>70 mJy), for which $150 \mathrm{MHz}$ fluxes are available from the $7 \mathrm{C}$ survey to quantify the $150 \mathrm{MHz}$ flux attenuation as a function of distance from the image phase center. We derived a function for the primary beam attenuation (see Figure 5) and we used it to correct the Lofar source fluxes. In Figure 6 we show the result of this correction for six sources brighter than $100 \mathrm{mJy}$ for which radio spectra can be obtained using existing multi-frequency information (VLSS, 7C, WENSS, NVSS).

It is worth to note that the corrected Lofar $150 \mathrm{MHz}$ fluxes are in very good agreement with the $7 \mathrm{C}$ flux density measured at the same frequency. 


\subsection{Future work}

We will combine the $1.4 \mathrm{GHz}, 325$ and $150 \mathrm{MHz}$ Lockman Hole source samples with existing $610 \mathrm{MHz}$ data, in order to characterize the spectral index of the radio sources as a function of flux density, thereby probing the physical mechanisms that dominate this enigmatic radio population: optically thin (steep spectrum) or self-absorbed (flat spectrum) synchrotron emission. In addition we will search for ultra-steep radio sources generally associated to very high redshift galaxies. This analysis will be extended to sub-mJy and $\mu$ Jy sources as soon as deeper Lofar observations will become available. Existing multi-wavelength information, from optical to mid and far infrared, to X-ray bands, will allow us to classify the host galaxies, and derive redshifts, an essential step to better assess the physical and evolutionary properties of the mixed (AGN + SFGs) faint radio population.

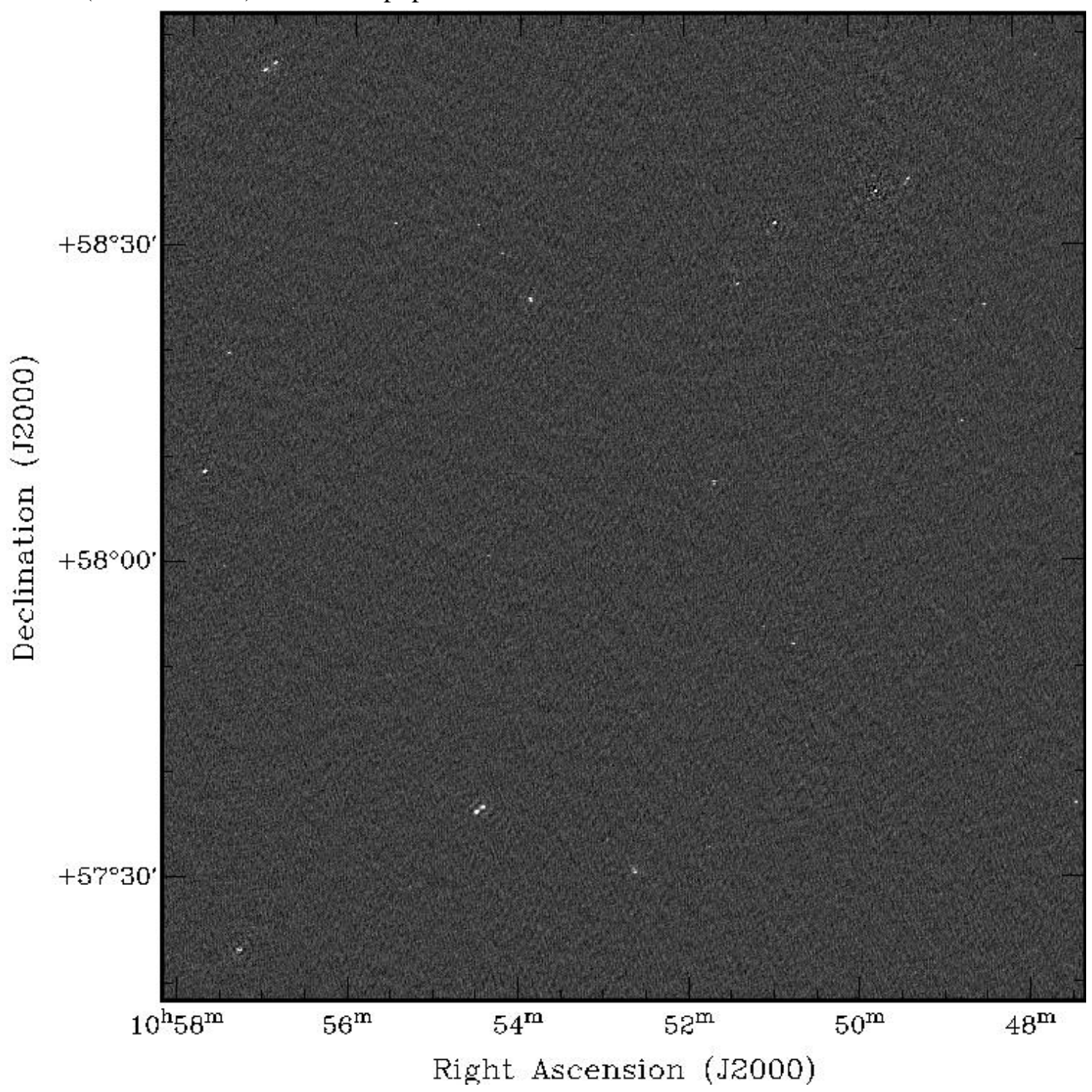

Figure 3 The inner part of the Lofar $150 \mathrm{MHz}$ image ( 2 sq. degr.). 


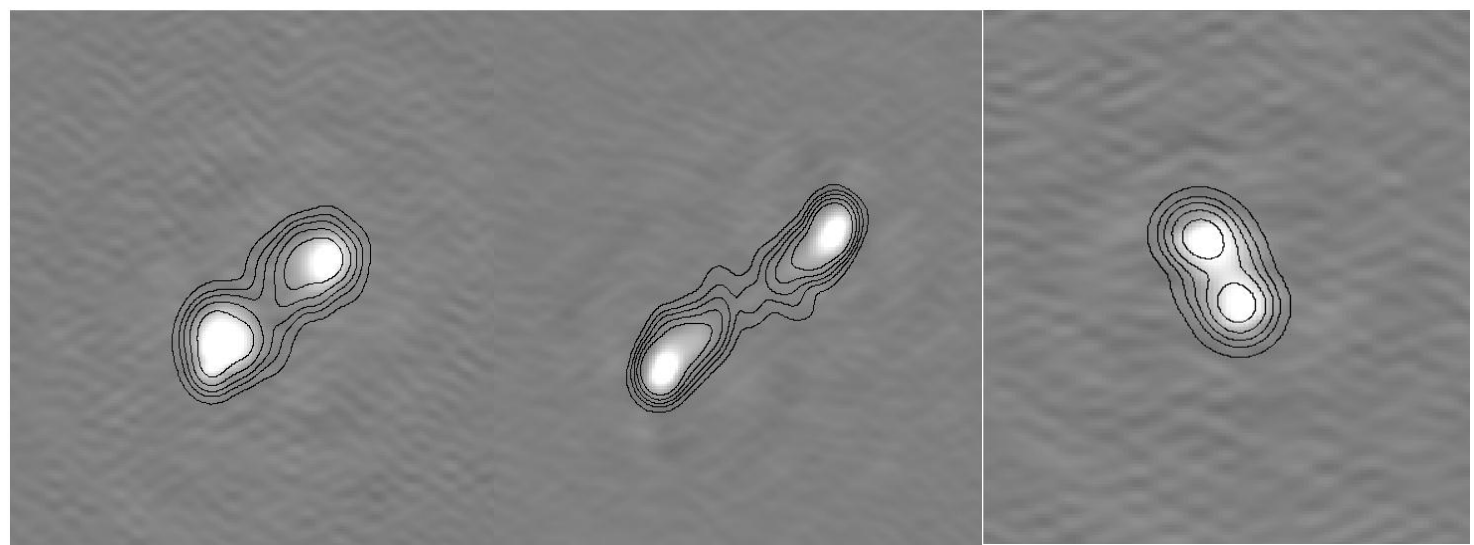

Figure 4 Three extended sources detected in the $150 \mathrm{MHz}$ Lofar image with $1.4 \mathrm{GHz}$ WSRT contours overlapped. The contours levels are 3,5,7,10,15 $\sigma$.

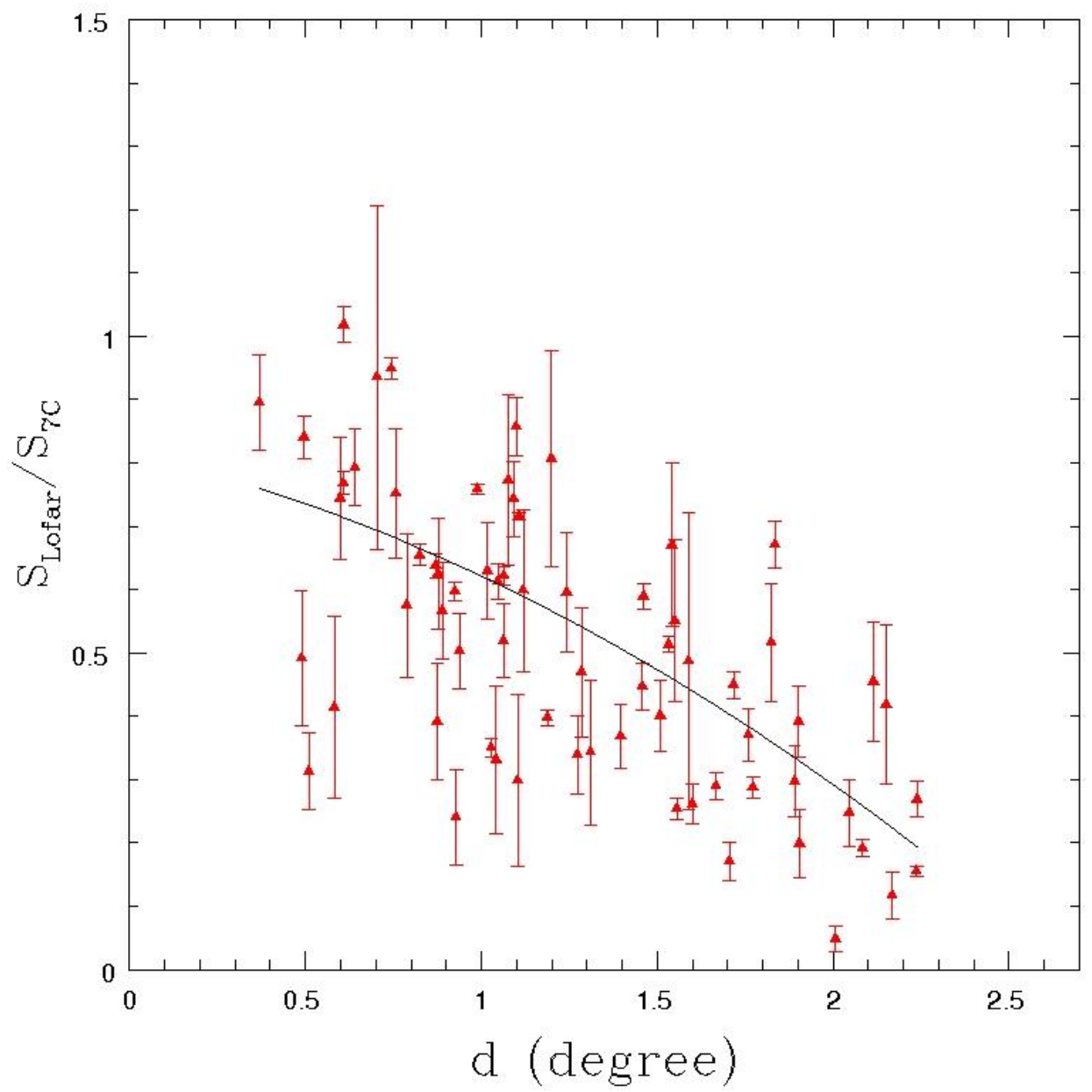

Figure 5 Lofar to 7 C flux ratio for the brightest sources in the Lockman Hole field. The solid line is a second order polynomial fit. 

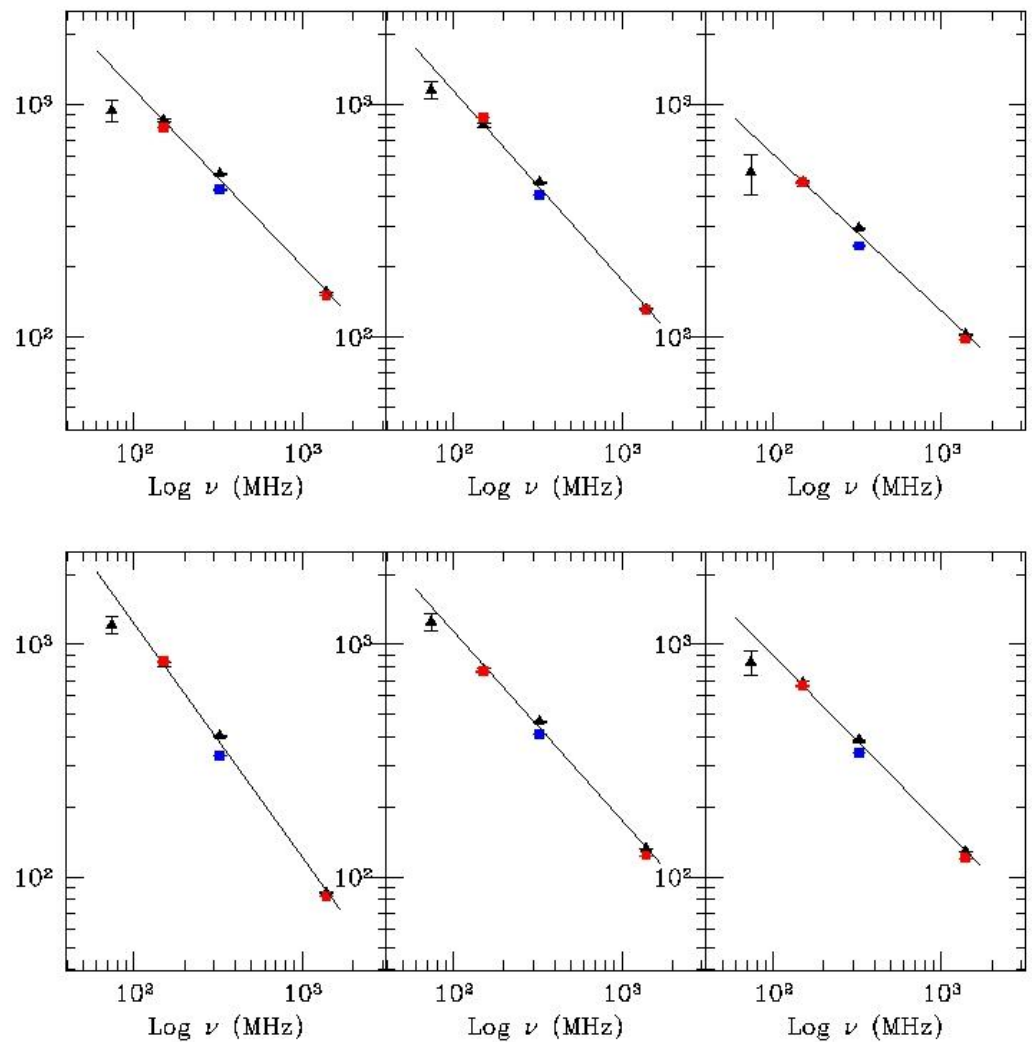

Figure 6 Flux density measurements for six bright sources in the Lockman Hole field. The black dots represent the flux measurements from NVSS (1.4 GHz), WENSS (325 MHz), 7C (151 MHz) and VLSS (74 MHz). The red dots represent the corrected Lofar (150 MHz) fluxes. Also shown in red the WSRT (1.4 GHz) fluxes obtained at similar resolution (9x11 arcsec). The blue dots represent the flux measurements from the WSRT $325 \mathrm{MHz}$ image, obtained at a resolution similar to that of the all-sky surveys $(40 x 70$ arcsec $)$.

\section{References}

Bondi M. et al., 2007, A\&A,463,519

Garn T., Green D. A., Riley J. M., Alexander P., 2008, MNRAS, 383,75

Magliocchetti M., Andreani P., Zwaan M. A., 2008, MNRAS, 383,479

Mignano, A.; Prandoni, I. ; Gregorini, L. et al., 2008, A\&A, 477, 449

Mitchell, K. J.; Condon, J. J. , 1985, AJ, 90, 1957

Prandoni I. et al., 2006, A\&A, 457, 517

Prandoni I.; de Ruiter H. R.; Ricci, R. et al. 2010, A\&A, 510, 42

Seymour N. et al., 2008, MNRAS, 386, 1695

Simpson C. et al., 2006, MNRAS, 372,741

Smolčić V. et al., 2008, ApJS,177,14

Windhorst, R. A.; Miley, G. K.; Owen, F. N.; Kron, R. G.; Koo, D. C., 1985, ApJ, 289, 494 